\title{
Abnormal neural integration related to cognition in schizophrenia
}

\author{
Dolan RJ, Fletcher PC, McKenna P, Friston KJ, Frith CD. Abnormal neural \\ integration related to cognition in schizophrenia. \\ Acta Psychiatr Scand 1999: 99 (Suppl. 395): 58-67. : Munksgaard 1999.
}

A striking feature of schizophrenia is the diversity of the phenomenology both within and between patients. This diversity can be contrasted with the well-circumscribed and stable deficits seen in classic neuropsychological syndromes. The argument will be advanced that the classic lesion model, based on the notion of a segregated deficit, is inappropriate in schizophrenia. Instead the idea will be developed that a more appropriate model is one derived from concepts of neural integration across large-scale brain networks. Empirical data derived from positron emission tomography (PET) within our laboratory that provide support for this suggestion will be presented. One critical observation from these data is a disruption of prefrontal-temporal interactions, under a variety of cognitive activation paradigms, in both chronic medicated and acute unmedicated schizophrenic patients. Furthermore, these data indicate that both regional and interregional neuronal function, including prefrontal-temporal interactions, can be significantly modulated by a neurochemical perturbation of ascending dopaminergic systems. The latter observations suggest that the deficit of abnormal cortico-cortical interactions are to some extent modifiable by neuromodulatory neurotransmitter systems.

\author{
R. J. Dolan ${ }^{1,2}$, P. C. Fletcher', \\ P. McKenna ${ }^{3}$, K. J. Friston', C. D. Frith ${ }^{1}$ \\ 'Wellcome Department of Cognitive Neurology. \\ Institute of Neurology, London, ${ }^{2}$ Royal Free Hospital \\ School of Medicine, London and " ${ }^{3}$ Fulborne Hospital, \\ Fulborne, Cambridge, UK
}

Key words: schizophrenia, cognition; psychopathology Raymond Dolan, Wellcome Department of Cognitive Neurology, Institute of Neurology, Queen Square, London WC1N 3BG, UK

\section{Introduction}

Schizophrenia is a multidimensional pathological disorder whose central characteristics include an early age of onset, a symptom profile that includes delusions, hallucinations, disordered thinking, impoverished action and, in many instances, a chronic deteriorating course (1). While a variety of neuropathological abnormalities have been reported in association with the disorder, the core pathophysiology remains elusive. The relapsing and remitting course and a responsiveness to pharmacological interventions all point to a disorder in which the critical disturbance involves a disorganization of brain function rather than a fixed pathology with an absence of function. In this respect the clinical phenomena in schizophrenia can be contrasted with the circumscribed and fixed psychological deficits seen in classical neuropsychological disorders that follow focal brain damage (2).

The value of functional imaging as an experimental tool for studying the pathophysiology of schizophrenia is to a large extent constrained by theoretical issues related to perspectives on brain function in general. Two theoretical frameworks inform current concepts of higher brain function. The classical view emphasizes processing in discrete modules, often anatomically circumscribed, and referred to as functional segregation. Put simply, this view assumes that localized regions of the brain are specialized for mediating unique psychological functions. Observations on the effects of focal brain lesions on language processing have provided the best-known support for theories of anatomically segregated function in the human brain $(3,4)$. The alternative perspective is the view that higher brain function is a property of interactions between functionally specialized, anatomically separate brain regions. The architectural arrangement of the human brain characterized by massive, and in most cases reciprocal, anatomical interconnectivity, provides a necessary substrate for cognitive processing that emphasizes integration across large-scale neural networks. The specification of the dense anatomical interconnectivity of the brain has coincided with the emergence of connectionist theories, first espoused in the last 


\section{Abnormal neural integration in schizophrenia}

century, that higher brain function involves cooperative interactions between anatomically separate brain regions $(5,6)$. This view derives much of its appeal from the likelihood that the function of segregated brain regions must be integrated during normal perception and action. A number of mechanistic accounts of how this integration might be realized have provided the focus for the elaboration of a range of connectionist theories (79). It is important to bear in mind that concepts of functional segregation and integration are not necessarily antagonistic, a fact that is highlighted by attempts to produce a unified framework for understanding brain function that explicitly acknowledges both perspectives (10).

How do concepts of brain function relate to functional imaging studies of schizophrenia? Functional imaging studies of schizophrenia are implicitly predicated on a perspective of functional specialization. In most of the published literature on schizophrenia the emphasis has been on identifying regional deficits in brain function through categorical comparisons of patients with appropriate control groups under conditions of either rest or activation. Since the pioneering work of Ingvar and Franzen it has been known that many schizophrenics show abnormally low resting blood flow in the frontal cortex (11). More recent studies show that this pattern is characteristic of patients with psychomotor poverty who show low blood flow, specifically in the left dorsolateral prefrontal cortex (DLPFC) (12). We would argue that a perspective of functional segregation, as applied in schizophrenia, is most usefully deployed in understanding the pathophysiology of discrete symptoms or syndromes. This viewpoint is congruent with observations that decreased DLPFC perfusion is not unique to schizophrenia, but is also found in depressed patients with psychomotor retardation (13). Furthermore, a symptom that is characteristic of the syndrome of psychomotor retardation, namely poverty of speech, is strongly predictive of decreased perfusion in this region, independent of whether patients are diagnosed as depressed or schizophrenic (14).

The focus of this chapter will be the development of an argument, derived from our own empirical investigations using functional neuroimaging, that a powerful framework for understanding schizophrenia is that derived from notions of functional integration. Specifically, it will be argued that a fundamental aspect of the pathophysiology of schizophrenia is a disturbance in functional integration between anatomically diverse brain regions that manifests most profoundly in the context of cognitive activation.

\section{Measuring functional integration using functional imaging}

In normal brain function the specialized processing that occurs in anatomically diverse brain regions during, for example, processing of object identity or location, must be integrated to enable coherent perception and action. Integration of function has considerable theoretical importance in the study of the psychoses, where abnormal neural interactions is proposed as a fundamental pathophysiological mechanism (5). The measurement of aspects of this integration is a major challenge for functional neuroimaging. Two generic approaches have been described that provide a framework within which questions concerning functional integration can be addressed $(15,16)$. These approaches, based upon the analysis of functional and effective connectivity, adopt perspectives akin to those used in electrophysiological studies based on coherence analysis of multi-unit recordings of separable neuronal spike trains $(17,18)$. The simplest approach seeks to determine how brain activity in different brain regions, evoked by a cognitive task, covaries as a function of time. Distributed responses of this kind can be captured by measures of functional connectivity, which is defined as the temporal correlation between two or more regions of task-related neural activity (15). This approach is primarily descriptive, with the resulting eigen images or spatial modes identifying brain regions that represent different systems by virtue of their functional interactions. The other approach is referred to as effective connectivity, which - in contrast to functional connectivity - is mechanistic. With measures of effective connectivity the issue to be resolved is the effect on a brain region of one or more extrinsic inputs to that region. This is modelled in terms of the changes in a region as a linear sum of changes at all other points in the brain. The weighting of any target region to the one in question is a measure of the strength of that connection or its effective connectivity. Measures of effective connectivity rely upon an explicit theoretical model which may be linear or nonlinear (19). Effective connectivity has been used to measure the mutual influence of $\mathrm{V} 1$ and $\mathrm{V} 2$ in human subjects based upon data from fMRI (16). The potential applications of effective connectivity are extremely wide ranging, and include assessment of changes in the interaction between brain regions that may occur as a function of learning or alteration in a modulatory neurotransmitter inputs to a region. Measures of effective connectivity are likely to assume increasing importance in future studies of the psychoses. However, up to the present time approaches to studying the pathophysiology of schizophrenia derived from a perspective 


\section{Dolan et al.}

of neural integration have relied solely on measures related to functional connectivity.

\section{Functional integration in chronic schizophrenia}

The utility of approaches to exploring the pathophysiology of schizophrenia based on functional connectivity was first demonstrated in the context of studies of chronic schizophrenia. Here we studied an aspect of response generation, using a paced word fluency task, in three separate groups of patients with chronic schizophrenia. There are two basic forms of these tasks that involve either generating many words in a particular category (e.g. words beginning with F) or generating one word that is linked to a cue word (e.g. generating verbs for nouns, cake - eat) $(20,21)$. Although the tasks have different qualities, the pattern of brain activity observed in normal subjects is virtually identical, involving activation of the left DLPFC and the anterior cingulate cortex and deactivation of posterior brain regions including the superior temporal cortices. The schizophrenic patients studied with regard to this task were allocated to separate groups on the basis of performance on the verbal fluency task before the scan session. Group 1 consisted of poor performers on the task in terms of the number of items produced. Group 2 consisted of patients who produced odd or inappropriate responses to the task. Group 3 consisted of patients who produced normal responses to the task. The control group consisted of six age- and sex-matched normal subjects. During the study, patients and controls produced verbal responses every $5 \mathrm{~s}$ to a target letter, or repeated a cue word in a simple repetition control task. The paced form of the task ensured that patients produced the same number of words as controls during the scan, and avoided the problem of performance differences between groups, which can make interpretation of functional imaging studies of abnormal groups problematic. For example, if group comparisons are associated with performance differences, then it is difficult to assign abnormal neural activation to some aspect of the pathophysiology of the disorder or the abnormal task performance.

In the group of 18 chronic schizophrenic patients as a whole we found no differences in the magnitude of the activations in DLPFC or anterior cingulate cortex with respect to controls (22). However, using an eigen image analysis which captures the main profiles of correlated activity expressed during task performance, striking differences in the overall response profiles were observed between the patients and the control subjects. In normal subjects the task-dependent profile, which accounted for most of the variance in the data, showed left dorsolateral prefrontal and anterior cingulate activation and bitemporal, medial prefrontal and posterior cingulate deactivation. In the three schizophrenic groups this pattern of taskdependent correlated activation was absent, and instead the main profile consisted of both dorsolateral prefrontal and also temporal activation (as opposed to deactivation). In other words, there was a striking failure to demonstrate the normal pattern of reduced activity seen during verbal fluency tasks in the left superior temporal cortex (23). An important aspect of the data was the fact that this abnormal pattern was expressed in the three schizophrenic groups who differed on verbal fluency task performance outside the scan, and who also showed profound differences in symptom expression of their illness. In other words, the abnormal pattern related to the fact of having the illness itself, rather than to a particular form of its expression.

\section{Functional integration in schizophrenic patients with memory impairment}

The importance of this finding of abnormal functional integration necessitates an attempt to replicate the basic finding in a further sample of patients. In a subsequent study we employed a study design which examined abnormal frontotemporal interactions using a different cognitive task. This study involved two groups of schizophrenic subjects, with and without significant memory impairment, and a reference control group. The designation of memory impairment was ascertained outside the context of the study, and was based on subjects' performance on the Rivermead Behavioural Memory Test (RBMT) (24).

For the cognitive activation we used an episodic memory task that involved listening to, and immediately recalling, auditorially presented word-lists. Memory load, defined on the basis of list length, was systematically varied across scans. This parametric design ensured that even in the most impaired schizophrenic subjects performance comparability would be present across the two groups of schizophrenic and control subjects for at least some of the scans. This parametric variation also enabled an analysis of the ways in which activity in different brain regions altered with changing memory demands of the task (25). In the context of the study, words were presented auditorially at a rate of 1 every $2 \mathrm{~s}$, and retrieval was placed by the experimenter counting at the same rate. Learning and retrieval of the same list 
went on throughout the scan. Across the 12 scans, lists varied in length from 1 to 12 items inclusive, and the order was pseudo-randomized across subjects. Thus, for example, during the first scan, a subject might repeatedly hear and retrieve a 7item word-list, during the second scan a 3-item list, during the third scan a 12-item list, and so on. Performance for each list presentation was recorded, and the same number of words in total was presented across each scan condition.

To limit the relevant comparisons to regions that are important with regard to memory function, we confined the analysis of group differences to regions which had shown memory-related activations when the three groups were analysed in combination. Similarly, the analysis of differences in deactivations was confined to those regions which had shown memory-related deactivations when the three groups were analysed in combination. Essentially, this 'masking' of comparisons constitutes a statistically more rigorous approach since, by confining analysis to a subset of voxels (defined by an orthogonal comparison), the likelihood of false positives is reduced.

The pattern of memory-load-related activations encompassed areas that would be predicted on the basis of our previous work on memory, and included the bilateral prefrontal cortex and posterior parietal cortex. Task-related deactivations were seen in temporo-parietal regions bilaterally. Comparisons of memory-related activations in the control group with those in the unimpaired schizophrenic subjects showed no significant differences. However, there were significant failures of memory-related deactivations in the region of the post-central gyrus/superior temporal gyrus bilaterally. For the comparisons between the control group and the impaired group, a significantly greater activation was seen in the schizophrenic group centred on the left inferior parietal lobe. Failure of deactivation was seen in the post-central gyrus/superior temporal gyrus bilaterally and in the medial prefrontal cortex. The direct comparison of the two schizophrenic subjects showed a relative failure of deactivation in the post-central gyrus/ superior temporal gyrus bilaterally and in the medial prefrontal cortex in the memory-impaired group.

This study provides a striking replication of the core observation described in our original study of abnormal prefrontal interactions with posterior brain regions during cognitive activation in schizophrenic patients. The replication of the finding is important in a number of respects. First, the abnormal pattern of prefrontal and temporal interactions is expressed in this study under different task conditions (memory-related activation as compared to verbal fluency in our original observation), which provides evidence that the finding is not cognitive domain or task specific. Secondly, the patient groups selected on different clinical criteria from those in our original study again expressed this same pattern of abnormality. The two studies combined indicate that a common pattern of pathophysiological abnormality in schizophrenia relates to a disturbance of patterns of integration between prefrontal and posterior brain regions during cognitive activation. Furthermore, there is the suggestion in the comparison of memory-impaired with nonmemory-impaired patients that the more severe the clinical expression of the disorder, the greater the disturbance in the functional interactions between brain regions. A cautionary caveat in relation to these findings is that both studies are based on observations in chronic patients with end-stage disease. Furthermore, all of the patient groups studied were on chronic neuroleptic medication. One possible explanation for these findings is that they represent either the effects of the evolutionary stage of the disorder or the effect of chronic neuroleptic use. The former interpretation can be addressed by studying patients in the acute phase of illness, while the latter issue can be addressed by studying drug-free patients.

\section{Neuromodulatory effects of dopamine neurotransmission on cognition}

In the context of observations of abnormal corticocortical integration in patients with dopamine blockade, it is also important to consider the general issue of the effect of dopamine neurotransmission on cortical function. Modulatory effects of dopamine on glutaminergic inputs to pyramidal cells in the prefrontal cortex can be demonstrated in animals (26-28). These effects on prefrontal cortical function would appear to be mediated via the $D_{1}$ receptor (29). Overall, the net effects of dopamine on the firing of pyramidal cells are inhibitory $(30,31)$. However, it is the effect of dopamine perturbations on task-related neuronal activity, rather than the spontaneous or baseline firing rates of target neurones, that provides the most meaningful framework for understanding the role of dopamine in cortical processing. The term neuromodulation is used to specify situations whereby the main effects of a neurotransmitter involve modifying the impact of other synaptic or non-synaptic inputs on common postsynaptic target cells (32). Thus the most important postsynaptic effects of dopamine are evident through alterations of the effectiveness of other excitatory or inhibitory 


\section{Dolan et al.}

synaptic inputs to target brain systems that mediate specific cognitive operations (33-35).

Single-cell recordings from the prefrontal cortex of monkeys, studied while performing delayed response tasks, have shown a differential cellular response to the micro-iontophoretic application of dopamine. A subpopulation of prefrontal cells, which increase their firing rate during a delay period, are further enhanced by dopamine manipulation, while the activity in cells that are not engaged by the task either remains unaltered or is inhibited (36). These effects can be conceived of as altering the state of a network subserving a specific psychological function by increasing task-specific firing, relative to background firing, in specific populations of prefrontal cells. The effects of neuroleptics on patterns of cellular activation have highlighted the receptor-mediated mechanisms that underlie such a differential response. Haloperidol and fluphenazine both anatagonized task-related dopamine augmentation of cell firing, but not the selective $\mathrm{D}_{2}$ antagonist sulpiride. These observations suggest that a $D_{1}$ effect is responsible for dopamine-mediated altered task-related neuronal activity in prefrontal neurones (37). A recent ex-situ study on human cortex has demonstrated a dopamine facilitation of NMDA ( $\mathrm{N}$-methyl-Daspartate) excitatory amino-acid receptor action, an effect that seems to be mediated by the $D$, receptor $(38,39)$.

In terms of neurophysiological effects it seems that activation of dopamine $D_{1}$ receptors, in particular, may be related to the performance of delayed response tasks where the underlying processes involve the temporal organization of behaviours guided by short-term memory (37). Local injections of selective $D_{1}$ antagonists, such as SCH23390 and SCH39166, directly into the prefrontal cortex of rhesus monkeys induce errors and increased latencies on an oculomotor task that requires memory-guided saccades. The drugs had no effect on performance in a task that required visually guided saccades, indicating that sensory and motor functions were unaltered. Thus $D_{1}$ receptors have a permissive role in the mnemonic or predictive functions of the primate prefrontal cortex (40). Recent non-human primate experiments that combine iontophoretic analysis of dopamine receptors with single-cell recording during behaviour have produced a refined analysis of dopaminergic effects on prefrontal cortical neurones (41). In an oculomotor delayed-response (ODR) paradigm that requires the animal to remember the spatial position of a stimulus, the iontophoretic application of a low dosage of the selective dopamine antagonist $\mathrm{SCH} 39166$ enhanced activity in neurones that showed maximal firing during the delay period between the cue and the motor response. This enhancement of the neuronal response could be reversed by the iontophoretic application of the $\mathrm{D}_{1}$ partial agonist SKF 38393. These effects were specific to taskdependent units, and no effects were observed on the general excitability of the cells studied. At higher dosages the same $\mathrm{D}_{1}$ antagonist inhibited prefrontal unit firing in a non-specific fashion, as did the $\mathrm{D}_{2}$ antagonist raclopride, with an effect that was evident not only in the memory field cells but also in non-memory-task neurones (41). The specificity of the $D_{1}$-mediated effects implies that blockade disinhibits specific excitatory inputs to the same cell. The overall findings can be interpreted as being consistent with a proposal that there is an optimal level of $D_{1}$ receptor-mediated activity for memory-related neuronal function. A lack of dopamine inputs or an excess of dopamine can therefore have similar functional effects on task-related cell firing.

\section{Dopamine neuromodulation in acute unmedicated schizophrenic} patients

In view of strong neurobiological evidence that dopamine has a regulatory role in cortical function, we chose to study patterns of neuronal activation engendered by a cognitive task, in normal and schizophrenic subjects, under two conditions involving the presence or absence of a dopamine challenge $(42,43)$. The decision to study dopamine was based on the previously discussed considerations and by the suggestion of a dopaminergic abnormality as an important mediating mechanism in the disorder (44). For example, there is evidence for psychosis-inducing effects of dopamine agonists (45) and antipsychotic effects of dopamine antagonists which remain the principal therapeutic mechanism of all antipsychotic drugs (46-48). Direct evidence for an abnormality in dopamine function has been lacking, and in-vivo, quantitative PET studies of dopamine receptor numbers have been inconclusive (49-51). However, functional imaging provides evidence of increased dopamine function in schizophrenic patients $(51,52)$, coupled with complementary evidence of increased central dopa decarboxylase activity $(9,52)$.

The type of experimental design that we adopted constituted an example of a psychopharmacological activation paradigm in which we have previously demonstrated prefrontal neuromodulatory effects of dopamine perturbations $(53,54)$. As we are primarily interested in the neuromodulatory effects of dopamine, we first engender a cognitive activation and then examine the effects of altering 


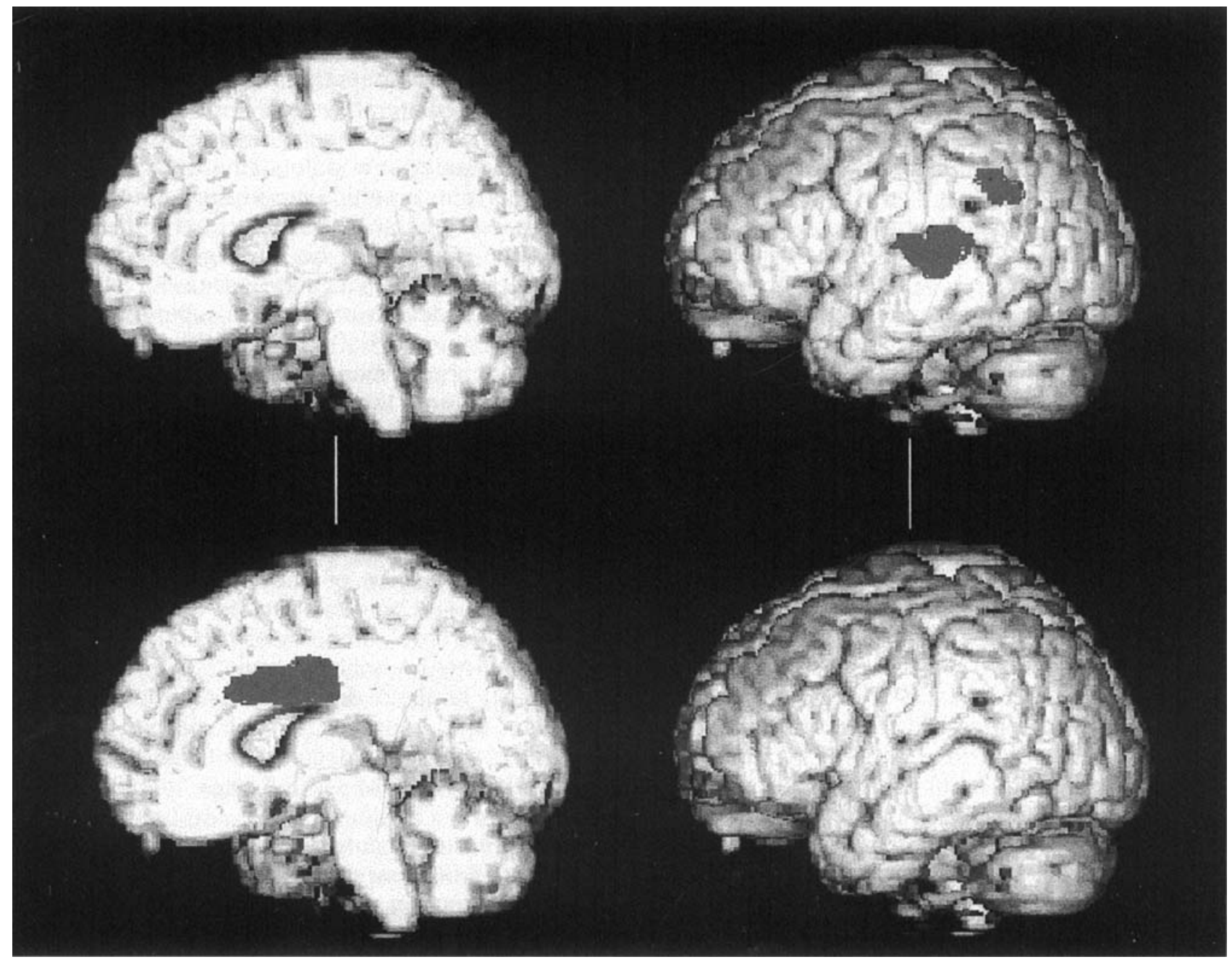

Fig. 1. Statistical parametric maps (SPMs) rendered on to spatially transformed magnetic resonance image scans. The SPMs are derived from comparisons between groups of acute unmedicated schizophrenic subjects and healthy controls performing verbal fluency tasks. The upper panel shows medial and lateral views of the right and left hemispheres, respectively. It indicates a relative failure in the schizophrenic group of anterior cingulate cortex activation (top left) and overactivation of the superior temporal cortex (i.e. failure of deactivation) (top right) during verbal fluency. The lower panel shows the same comparisons after administration of apomorphine (as described in the text), and now demonstrates a relatively greater activation of anterior cingulate cortex in the schizophrenic subjects compared to controls, with a normalization of the superior temporal pattern of activity.

dopamine neurotransmission on the pattern of activation that ensues. This type of paradigm also dictated that the patients we studied should not be receiving concurrent neuroleptic medication. Consequently, we recruited 12 right-handed subjects who fulfilled DSM-III-R criteria for schizophrenia from hospitals, out-patient departments and general practitioners in London. The mean age of these patients was 26 years (SD 7 years), and they had a mean duration of illness (as estimated from personal interview, interviews with relatives and medical records) of 4.3 years (SD 6 years). All of the patients had been free of neuroleptic medication for at least 6 months and, of the 12 subjects, nine were neuroleptic-naive. As controls we recruited 12 healthy, age-matched male subjects who did not have a past history of psychiatric or neurological illness. For the cognitive challenge we again used a paced orthographic verbal fluency task in which a chosen letter was repeated orally by the experimenter at a rate of once every $5 \mathrm{~s}$. Subjects were required to produce verbally a word beginning with that letter for each repetition. If a subject was no longer able to produce a response for a given letter, he was instructed to say 'pass' and the experimenter switched to a different letter. The control condition, also performed three times and alternating with the experimental condition, was a paced word repetition task that involved the same number and frequency of cues and responses as the verbal fluency task. As this was a psychopharmacological study, 6 subjects from each of the two groups (the schizophrenic and control groups) were given apomorphine (10 mcg kg-1 subcutaneously) 


\section{Dolan et al.}

after the second scan. After a further $10 \mathrm{~min}$, scanning was resumed and the last four scans were obtained under the condition of this dopamine challenge. As a control, the other 6 subjects in the schizophrenic and control groups were given a subcutaneous placebo injection (water) after their second scan.

We initially examined activations associated with the verbal fluency task $(42,43)$. The relevant analysis was confined to the non-apomorphine scans, since a cognitive task-associated activation in the presence of apomorphine incorporates an interaction with the drug. In the control subjects, the verbal fluency task was associated with activation of the following areas: the prefrontal cortex bilaterally, the anterior cingulate cortex and the thalamic/sub-thalamic regions. In the schizophrenic subjects there was also task-associated activation in the prefrontal cortex bilaterally and the anterior cingulate gyrus. In addition, activations were seen in the left inferior parietal lobe, the left superior temporal gyrus and the posterior cingulate gyrus. In terms of task-related deactivations in the control subjects, there were relative decreases in rCBF in the fluency compared to the control condition centred on the superior temporal gyri bilaterally and the posterior cingulate gyrus. Group differences in cognitive task-related activations and deactivations were determined by an analysis of all of the non-apomorphine scans. This group comparison revealed two critical differences, consisting of a relative failure of activation in schizophrenia in the anterior cingulate cortex and a relative failure of deactivation in the left superior temporal gyrus and the left inferior parietal lobe.

Having demonstrated striking group differences in the pattern of elicited activation and deactivation, we next explored the functional effects of apomorphine on these overall patterns of activation. These analyses are most appropriately framed in the context of whether there were group-specific task-induced effects which were modulated (either attenuated or augmented) by apomorphine. This can be determined by comparing patterns of predrug activations and deactivations with those that occur post drug both within and between groups. Pre- and post-placebo scans were included in the analysis, as described above, to remove order effects (i.e. we looked for a three-way interaction relative to placebo). This between-group comparison of the apomorphine-related modulations of cognitive-task-related activity was limited to a subset of voxels identified by the differential verbal fluency activations between the two groups. This preselection represents a test of two independent hypotheses, namely that the area is differentially activated in schizophrenia and that this differential activation is modulated by apomorphine. This comparison showed that there was a significant augmentation of the task-related anterior cingulate activation in schizophrenic subjects, and an attenuation of task-related activity in the left superior/middle temporal gyrus. The findings from this study are illustrated in Fig. 1.

This study of acute unmedicated patients indicated the presence of both a segregated abnormality in the anterior cingulate cortex and a failure of task-related deactivation in the superior temporal cortex. Strikingly, both abnormalities were modulated by apomorphine such that, following its administration, there was enhanced cingulate activation and a relative normalization of superior temporal deactivation. The findings can be interpreted as indicating that, in schizophrenia, the pathophysiology reflects processes characteristic of disorders of segregation and integration. The regional specificity of the finding of abnormal activation and modulation is consistent with evidence from other sources for a cingulate abnormality in schizophrenia. Core cingulate pathology has been described in neuropathological studies that report neuronal deficits, loss of interneurones and a decrease in $\mathrm{GABA}_{\mathrm{a}}$ receptors indicative of loss of GABA-ergic inhibitory cells (55-58).

The anterior cingulate cortex has been ascribed a number of different specialized functions, including a role in attention. Although attention is ill defined, one key aspect is captured by the notion of allocation of resources to activities that are currently most salient. At the neural level this allocation might be achieved through a modulatory influence on processing systems that are competing for limited processing resources. Activation of the anterior cingulate is a common finding in PET studies where the common cognitive component involves engaging selective attention on a stimulus or task and the inhibition of inappropriate, competing responses $(20,59,60)$. Lesions to this region are also associated with a wide range of neuropsychological disorders, including aberrant social behaviour, akinetic mutism, diminished selfawareness and depression (61). Its widespread connections and functional heterogeneity (61) suggest a role in the regulation or co-ordination of activity within interconnected brain areas.

Can the findings be interpreted in terms of a single unifying framework? One possibility is that the anterior cingulate cortex has a role in modulating neuronal interactions between diverse brain regions in the context of cognition. In the case of acute schizophrenia, there is a breakdown in this modulatory function which manifests as both a regional deficit (a failure of task-elicited cingulate 
activation) and abnormal prefrontal temporal interactions (inferred from the failure of superior temporal deactivation). In other words, the perspectives of abnormalities with respect to segregation and integration both apply. That this disruption of the modulatory influences of the anterior cingulate cortex might be a plausible mechanism in schizophrenia can be inferred from two lines of evidence. First, there is the phenomenon of re-afference copy, which has been invoked as an explanation for the coherent neural integration that maintains a stable representation of the world when, for example, we move our eyes (62). In this situation the retinal image is changing, yet we experience a stable world. Robinson and Wurtz (1976) have proposed that the source of modulation in this situation is the frontal eye fields (FEF), which results in a differential response in cells in the superior colliculus to moving stimuli but not to the eye moving across a stationary target (63). Other examples of this type of context-specific modulation include the differential response of units within the superior temporal cortex to external as opposed to self-generated vocalizations.

Empirical data that capture this putative modulatory influence of the cingulate come from our own studies of episodic memory encoding under dual-task conditions. When subjects were required to listen and remember a list of word pairs while simultaneously performing either a difficult or an easy motor distracting task, we noted an attenuation of left prefrontal activation under the condition of simultaneous performance of the difficult distracting task. This attenuation in left DLPFC activity was associated with a significant augmentation in the response of the anterior cingulate cortex. These data are consistent with the role of this structure in mediating attention to action, and suggest that this role is mediated by its modulatory effects on regions that are competing for limited attentional resources.

How can these issues be related to our finding of dopaminergic modulation of anterior cingulate function in schizophrenia? One critical question related to this is whether there is evidence that apomorphine influences the phenomenology of the condition. The most relevant finding in this context is the observation that apomorphine, at a similar dosage to that used in our studies, can produce an acute amelioration of schizophrenic symptoms (64). Attentional deficits are also core abnormalities in schizophrenia $(65,66)$. One interpretation of the behavioural and neuromodulatory effects of apomorphine is that schizophrenia does indeed involve a hyperdopaminergic state which exerts a net inhibitory effect on task-related activations in the cingulate. As apomorphine, at the low dose used in the present study, has a predominantly presynaptic effect, this would result in a functional decrease in dopamine neurotransmission (33). The end result would be a release from the tonic inhibitory effects of dopamine, which would be consistent with our observation of an associated post-drug increase in task-related neural activity. In our previous studies of chronic schizophrenic subjects receiving dopamine antagonists we found normal anterior cingulate activation in the context of an identical verbal fluency task (22), and this is consistent with the suggestion that dopamine blockade can normalize cingulate function.

\section{Conclusions}

The idea that the core pathology in schizophrenia relates to a disturbance in the integration of neural activity across brain regions during cognition has been developed using empirical data from functional neuroimaging. Strikingly, we have replicated a finding of abnormal prefrontal and superior temporal interactions in six separate groups of acute and chronic schizophrenic patients. The underlying basis for this abnormal connectivity is not known, but we note that it is consistent with a number of well-articulated theories of schizophrenia that involve genetic, neurodevelopmental and neurochemical mechanisms or combinations thereof. Although our studies have highlighted a disturbance in neural interactions in schizophrenia, we have also indicated an important role for dopamine in the regulation of cingulate activity. We suggest that the functional role of dopamine in the cingulate may be to facilitate integration of information-processing through an attentional modulation of cortico-cortical integration. Thus a disturbance in dopamine modulation of cingulate function could impair the regulation of cortical neural networks and provide a framework for reconciling theories of neurochemical abnormalities with those that suggest abnormal corticocortical interactions in schizophrenia.

\section{References}

1. Andreasen NC, Carpenter WT Jr. Diagnosis and classification of schizophrenia. Schizophr Bull 1993;19:199-214.

2. FrITH CD. The cognitive neuropsychology of schizophrenia. Hove: Lawrence Erlbaum, 1992.

3. Broca P. Sur le siege de la faculte du langage articule. Bull Soc Anthropol 1865:6:377-396.

4. Wernicke: C. Der Aphasische Symptomenkomplex. Breslau, Poland: M. Cohn and Weigert, 1874.

5. Wernicke C. Grundrisse der Psychiatrie. Leipzig: Thieme, 1906.

6. Goltz F. Transactions of the Seventh International Medical Congress. Vol. 1. London: J. W. Kolkmann, 1881. 


\section{Dolan et al.}

7. Singer W. Search for coherence: a basic principle of cortical self-organisation. Concepts Neurosci 1990;1:5-54.

8. ECKhORN R, BAUer R, JORIDAN $W$ et al. Coherent oscillations: a mechanism of feature linking in visual cortex? Biol Cybernetics 1988;60:121-130.

9. Gray CM, Konig P, Engel AK et al. Oscillatory responses in cat visual cortex exhibit inter-columnar synchronization which reflects global stimulus properties. Nature 1989;338:334-337.

10. Tononi G, Sporns O, Euelman GM. A measure for brain complexity: relating functional segregation and integration in the nervous system. Proc Natl Acad Sci USA 1994;91:5033-5037.

11. Ingvar DH, Franzen G. Abnormalities of cerebral blood flow distribution in patients with chronic schizophrenia. Acta Psychiatr Scand 1974;50:425-462.

12. Liddle P, Friston KJ, Frith CD et al. Patterns of cerebral blood flow in schizophrenia. Br J Psychiatry 1992;160:179186.

13. Dolan RJ. Functional imaging in the neurobiology of the psychoses. Semin Neurosci 1995;7:165-171.

14. DOLAN RJ, BEnCH CJ, Liddle PF et al. Dorsolateral prefrontal cortex dysfunction in the major psychoses; symptom or disease specificity? J Neurol Neurosurg Psychiatry 1993;56:1292-1298.

15. Friston KJ, Frith CD, Liddle $P$ et al. Functional connectivity: the principal-component analysis of large (PET) data sets. J Cereb Blood Flow Metab 1993;13:5-14.

16. Friston KJ, Ungerleider LG, Jezzard $P$ et al. Characterizing modulatory interaction between areas $\mathrm{V} 1$ and $\mathrm{V} 2$ in human cortex: a new treatment of functional MRI data. Hum Brain Mapping 1995;2:211-224.

17. Gerstein GL, Perkel DH. Simultaneously recorded trains of action potentials: analysis and functional interpretation. Science 1969;164:828-830.

18. Gerstein Gl, Bedenbaugh P, Aersten AMHJ. Neuronal assemblies. Trans Biomed Engin 1989;36:4-14.

19. Friston KJ, Frith CD, Frackowiak RSJ. Time-dependent changes in effective connectivity measured with PET. Hum Brain Mapping 1993;1:69-79.

20. Frith CD, Friston KJ, LidDle PF et al. A PET study of word finding. Neuropsychologia 1991;29:1137-1148.

21. Posner Mi, Petersen SE, Fox PT et al. Localization of cognitive operations in the human brain. Science 1988;240:1627-1631.

22. Frith CD, Friston KJ, Herold $S$ et al. Regional brain activity in chronic schizophrenic patients during the performance of a verbal fluency task. $\mathrm{Br} \mathrm{J}$ Psychiatry 1995;167:343-349.

23. Friston KJ, Frith CD, Fletcher P et al. Abnormal frontotemporal interactions in schizophrenia. In: WATson SJ, ed. Biology of schizophrenia and affective disorders. New York: Raven Press, 1996:421-449.

24. Wilson B, Cockburn J, Baddeley A. The Rivermead Behavioural Memory Test manual. Bury St Edmonds: Thames Valley, 1985.

25. Grasby PM, Frith CD, Friston KJ et al. A graded task approach to the functional mapping of brain areas implicated in auditory-verbal memory function. Brain 1994;117:1271-1282.

26. ChIOdo LA, Berger TW. Interactions between dopamine and amino acid-induced excitation and inhibition in the striatum. Brain Res 1986;375:198-203.

27. Knapp AG, Dowling JE. Dopamine enhances amino acidgated conductances in cultured retinal horizontal cells. Nature 1987;525:437-439.

28. Pralong E, Jones RSG. Interaction of dopamine with glutamate- and GABA-mediated synaptic transmission in the rat entorhinal cortex in vitro. Eur $\mathrm{J}$ Neurosci 1993;5:760-767

29. Sawaguchi T, Goldman-Rakic PS. The role of D. dopamine receptor in working memory: local injections of dopamine antagonists into the prefrontal cortex of rhesus monkeys performing an oculomotor delayedresponse task. J Neurophysiol 1994;71:515-528.

30. Bunney BS, Aghajanian GK. Dopamine and norepinephrine innervated cells in the rat prefrontal cortex: pharmacological differentiation using microiontophoretic techniques. Life Sci 1976;19:1783-1792.

31. Thierry AM, Jay TM, Pirot S et al. Influence of afferent systems on the activity of the rat prefrontal cortex: electrophysiological and pharmacological characterization. In: ThIERRY AM, ed. Motor and cognitive functions of the prefrontal cortex. Berlin: Springer-Verlag, 1994:5-50.

32. DismukES RK. New concepts of molecular communication among neurons. Behav Brain Sci 1979;2:409-448.

33. Brozoski TJ, Brown RM, Rosvold HE et al. Cognitive deficit caused by regional depletion of dopamine in prefrontal cortex of rhesus monkey. Science 1979;205:929-932.

34. Foote Sl, Freedman R, Oliver AP. Effects of putative neurotransmitters on neuronal activity in monkey auditory cortex. Brain Res 1975;86:229-242.

35. Arnsten AFT, Goldman-Rakic PS. Alpha -adrenergic $_{2}$ mechanisms in the prefrontal cortex associated with cognitive decline in aged nonhuman primates. Science 1985;230:1273-1276.

36. Sawaguch T, Matsumura M, Kubota K. Catecholaminergic effects on neuronal activity related to a delayed response task in monkey prefrontal cortex. $\mathbf{J}$ Neurophysiol 1990;63:1385-1399.

37. Sawaguchi T, Matsumura M, Kubota $K$. Effects of dopamine antagonists on neuronal activity related to a delayed response task in monkey prefrontal cortex. J Neurophysiol 1990;63:1401-1411.

38. Cepeda C, Buchwald NA, Levine MS. Neuromodulatory actions of dopamine in the neostriatum are dependent upon the excitatory amino acid receptor subtypes activated. Proc Natl Acad Sci USA 1993;90:9576-9580.

39. Cepeda C, Radisavluevic Z, Peacock W, Levine MS, BuchWald NA. Differential modulation by dopamine of responses evoked by excitatory amino acids in human cortex. Synapse 1992;11:330-341.

40. Sawaguchi T, Goldman-Rakic PS. D dopamine receptors in prefrontal cortex: involvement in working memory. Science 1991;251:947-950.

41. Williams GV, Goldman-Rakic PS. Modulation of memory fields by dopamine $D_{1}$ receptors in prefrontal cortex. Nature 1995;376:572-575.

42. Dolan RJ, Fletcher P, Frrth CD et al. Dopaminergic modulation of an impaired cognitive activation in the anterior cingulate cortex in schizophrenia. Nature 1995;378:180-182.

43. Fletcher PC, Frith CD, Grasby PM et al. Local and distributed effects of apomorphine on fronto-temporal function in acute unmedicated schizophrenia. J Neurosci 1996;16:7055-7062.

44. Randrup A, Munkvad I. Evidence indicating an association between schizophrenia and dopaminergic hyperactivity in the brain. Orthomol Psychiatry 1972;1:2-7.

45. Davidson M, Keefe RSE, MoHS RC et al. l-dopa challenge and relapse in schizophrenia. Am J Psychiatry 1987;141:934-938.

46. Seeman P, Lee T, Chau-Wong $M$ et al. Antipsychotic drug doses and neuroleptic/dopamine receptors. Nature 1976;261:717-719. 
47. Creese I, Burt DR, Snyder SH. Dopamine receptor binding predicts clinical and pharmacological potencies of antischizophrenic drugs. Science 1976;192:481-483.

48. Firrier D. Experiments on the brain of monkeys. Proc $R$ Soc Lond 1875;23:409-430.

49. Farde L, Wiesel FA, Stone-Etander $S$ et al. $\mathrm{D}_{2}$ dopamine receptors in neuroleptic-naive schizophrenic patients Arch Gen Psychiatry 1990;47:213-219.

50. Fardf L, Sedvall G, WIEsel FA et al. Brain dopamine receptors in schizophrenia: PET problems. Arch Gen Psychiatry 1988;45:599-600.

51. Wong DF, Wagner HN JR, Tunf. LE et al. Positron emission tomography reveals elevated $D_{2}$ dopamine receptors in drug-naive schizophrenics. Science 1986;234:1558-1563.

52. Buchis. C, Wisf RJS, Mummery CJ el al. Non-linear regression in parametric activation studies. Neuroimage $1996 ; 4: 60-66$

53. Friston KJ, Grasby P, Frith CD et al. The neurotransmitter basis of cognition: psychopharmacological activation studies using positron emission tomography. In: Exploring brain functional anatomy with positron tomography. Chichester: John Wiley \& Sons, 1991:76-92.

54. Grasby PM, Friston KJ, BenCH CJ et al. The effect of apomorphine and buspirone on regional cerebral blood flow during the performance of a cognitive task measuring neuromodulatory effects of psychotropic drugs in man. Eur J Neurosci 1992;4:1203-1212.

55. Bines FM, DAvioson J, Biris JE. Quantitative cytoarchitectural studies of schizophrenic cortex. Arch Gen Psychiatry 1986;43:31-35.

56. Bines FM, McSpARrin J, Bird ED et al. Deficits in small interneurons in prefrontal and cingulate cortex of

\section{Abnormal neural integration in schizophrenia}

schizophrenic and schizoaffective patients. Arch Gen Psychiatry 1991;48:996-1001.

57. Bfanes FM, Vincent Si, Alsterberg $G$ et al. Increased $\mathrm{GABA}_{a}$ receptor binding in superficial layers of schizophrenic cingulate cortex. J Neurosci 1992;12:924-929.

58. Akbarian S, Bunnfy WE Jr, Potrin SG et al. Altered distribution of nicotinamide/adenine dinucleotide phosphate-diaphorase cells in frontal lobe schizophrenics implies disturbances in cortical development. Arch Gen Psychiatry 1993;50:169-177.

59. Fletcher PC. Frith CD. Grasby PM et al. Brain systems for encoding and retrieval of auditory-verbal memory: an in vivo study in humans. Brain 1995:118:401-416.

60. Frith CD, Friston KJ, LiDdie PF el al. Willed action and the prefrontal cortex in man: a study with PET. Proc R Soc Lond B 1991:244:241-246.

61. ZIHL J, VON CRAMON D, MAI N. Selective disturbance of movement vision after bilateral brain damage. Brain 1983:106:313-340.

62. Sperry W. Neural basis of the spontaneous optokinetic response produced by visual inversion. J Comp Physiol Psychiatry 1950;43:482-489.

63. Robinson DL, Wurtz RH. Use of an extraretinal signal by monkey superior colliculus neurons to distinguish real from self-induced movement. J Neurophysiol 1976; 39:852-870.

64. TAmminga CA. Schizophrenic symptoms improve with apomorphine. Science 1978;200:567-568.

65. Bleuler E. Dementia praceox or the group of schizophrenias. New York: International Universities Press, 1950.

66. McGhie A, Chapman J. Disorders of attention and perception in early schizophrenia. Br $\mathrm{J}$ Med Psychol 1961;34:103-116. 